\title{
Modeling Coplanar Waveguide Structures Constructed of Ferromagnetic Metal ${ }^{*}$
}

\author{
Nicholas Cramer, student member, IEEE \\ Dept. of Physics, University of Colorado, Colorado Springs, CO, 80918, USA \\ David K.Walker, member, IEEE \\ National Institute of Standards and Technology, Boulder, CO, 80305-3328, USA
}

\begin{abstract}
We fabricate coplanar waveguide transmission lines with thin-film $\mathrm{Fe}_{0.2} \mathrm{Ni}_{0.8}$ conductors. These lines demonstrate a power attenuation of $10 \mathrm{~dB} / \mathrm{cm}$ at ferromagnetic resonance frequencies of 5 to $10 \mathrm{GHz}$ for applied magnetic fields less than $100 \mathrm{~m} T$ ( $1 \mathrm{kG})$. In addition, the phase of the transmitted wave can be tuned by about 20 degrees $/ \mathrm{cm}$ by adjusting an applied magnetic field. We present a simple model for the complex reflected and transmitted waves as a function of material characteristics, geometry, and applied field We model the skin effect influence on the transmission line circuit parameters, including the line impedance and propagation constant, by considering the full effects of the conductor permeability and conductivity.
\end{abstract}

\section{INTRODUCTION}

As communication applications progress to higher and higher operating frequencies, there is increasing demand for devices able to operate at these frequencies. Devices such as tunable filters and phase shifters have traditionally relied on ferrites such as YIG for their operation [1]. These devices function at, or close to, the ferromagnetic resonance (FMR) frequency, which is determined by material properties such as saturation magnetization $\left(M_{s}\right)$ and by applied magnetic field. Due to their relatively low $M_{s}$, ferrimagnets require large magnetic fields in order to increase the resonance frequency above a few $\mathrm{GHz}$.

Ferromagnetic metals such as $\mathrm{Fe}$ and permalloy (Py or $\mathrm{Fe}_{0.2} \mathrm{Ni}_{0.8}$ ) have much higher $\mathrm{M}_{\mathrm{s}}$ and therefore their resonant frequencies are higher for a given applied field. The major drawback of these ferromagnetic metals is their high electrical conductivity. Ferrimagnets are insulators and have been engineered for decreased dielectric loss. Applying ferromagnetic metals in this way is clearly impractical: Instead, there have been various studies of devices constructed with the ferromagnet incorporated into a transmission line's conductive elements. The theoretical treatment of such devices was explored by Schloemann and co-workers [2] and by Camley and Mills [3]. Previous studies have used a thin ferromagnetic film incorporated in the ground plane or signal conductor of a microstrip structure [4]-[6]. Our recent study [7] used a coplanar waveguide (CPW) constructed of Py conductors on a GaAs substrate.

\section{THEORY}

When modeling a line based on a magnetic dielectric, the task usually involves starting with a model that assumes perfect conductors and a relative permeability in the dielectric equal to unity. Next, the equations are modified to accommodate the complex, anisotropic permeability of the dielectric. As a final step, conduction loss in the conductors is added. In our case, the dielectric is nonmagnetic and all magnetic effects enter the model via the skin effect in the conductors.

Modeling the skin effect is a two-step process. First, we extract the non-magnetic skin effect and second we modify it with the proper magnetic contribution. Extraction of the skin effect is aided by modeling the inductance and capacitance per unit length ( $L$ and $C$, respectively) by,

$$
L=\mu_{r} \mu_{0} g
$$

and,

$$
C=\frac{\varepsilon_{r} \varepsilon_{0}}{g}
$$

where $\mu_{r}$ and $\mu_{0}$ are the relative and free-space permittivities, respectively, $\varepsilon_{r}$ and $\varepsilon_{0}$ are the relative and free-space permeabilities, respectively, and $g$ is an unitless geometric factor that accounts for the device geometry. If we assume a lossless line with $\mu_{r}=1$ (the usual

\footnotetext{
* Publication of the United States Government, not subject to U.S. Copyright.
} 
assumptions made in models of "normal" lines), we find that the impedance has the form,

$$
Z_{0}=\sqrt{\frac{g^{2} \mu_{0}}{\varepsilon_{r} \varepsilon_{0}}}=\frac{g \eta_{0}}{\sqrt{\varepsilon_{r}}},
$$

and hence $g$ can easily be found using equations in the literature for $Z_{0}$ [8]. Next, we recognize that $Z_{0}$ is a function of skin depth as well as the material properties, $\mu_{r}$ and $\varepsilon_{r} . Z_{0}$ is a function of $a$, the signal line width, $b$, the ground plane spacing, $t$, the conductor thickness, and $h$, the substrate thickness. These dimensions are defined in the cross section shown in Fig. 1. If we correct $a$ and $b$ for the skin effect, we can construct two equations-one for $Z_{0}$, the zero skin depth impedance, and another for $Z_{0}$, the finite skin depth impedance,

$$
Z_{0}=Z(a, b, t, h)
$$

and,

$$
Z^{\prime}{ }_{0}=Z(a-\delta, b+\delta, t, h) .
$$

From these two impedances, two $g$ 's can be extracted ( $g$ and $g$, respectively). Note that:

$$
g^{\prime}-g=\Delta g>0
$$

Now we propose that $\Delta g$ represents the added influence of the skin depth region of the conductors and therefore we can express the modified inductance $L$ ' as:

$$
L^{\prime}=\mu g^{\prime}=\mu_{\text {dielectric }} g+\mu_{e f f} \Delta g,
$$

where $\mu_{\text {dielectric }}$ is assumed to be $\mu_{0}$ and $\mu_{\text {eff }}$ is an effective permeability in the ferromagnetic conductor (a complex quantity). Note that for non-magnetic conductors $\mu_{e f f}$ is equal to $\mu_{c}(1+j)$, where $\mu_{c}$ is the dc permeability of the metal, and that this formulation reduces to common expressions for $L$ and $R$ that account for the skin effect [9].

The next task is to derive expressions for the skin depth and $\mu_{\text {eff }}$ First, note that $\mu$ in the conductor can be represented by the "Voigt permeability" $\left(\mu_{\text {voigt }}\right)$ [2],

$$
\mu_{\text {voigt }}=\frac{\mu_{1}^{2}-\mu_{2}^{2}}{\mu_{1}} \text {, }
$$

where,

$$
\mu_{1}=\mu_{0}+\frac{\mu_{0} \gamma_{0} M_{S}\left(\gamma_{0} B_{0}-j \Gamma \omega\right)}{\left(\gamma_{0} B_{0}-j \Gamma \omega\right)^{2}-\omega^{2}}
$$

and

$$
\mu_{2}=\frac{\mu_{0} \gamma_{0} M_{s} \omega}{\left(\gamma_{0} B_{0}-j \Gamma \omega\right)^{2}-\omega^{2}} .
$$

$B_{0}$ is the applied magnetic field, $\gamma_{0}$ is the gyromagnetic ratio, and $\Gamma$ is the damping factor. Schloemann [2] proposed that the complex scalar $\mu_{\text {voigt }}$, instead of the entire permeability matrix, be used to model the interaction of EM waves with ferromagnets. The relevance of this approach was rigorously confirmed later by Astalos and Camley [10]. Also note that $\mu_{\text {voigt }}$ is not $\mu_{e f f}$ the former only includes magnetic effects and the latter must include both magnetic and electric (conduction) effects.

The skin depth and $\mu_{e f f}$ both derive from the propagation constant of a wave in a ferromagnetic conductor:

$$
\gamma=j \omega \sqrt{\varepsilon \mu_{v o i g t}},
$$

where $\omega$ is the angular frequency. As is usual for a conductor,

$$
\varepsilon \approx \frac{j \sigma}{\omega}
$$

where $\sigma$ is the conductivity. Hence, the skin depth as a function of frequency and applied field can be found for a ferromagnetic conductor with known conductivity and magnetic properties:

$$
\delta=\frac{1}{\operatorname{Re}(\gamma)}
$$

Below, we derive an expression for $\mu_{e f f}$ using the propagation constant. First, we follow the usual technique for determining surface impedance using a known propagation constant; that is, we integrate the surface current density due to an electric field and divide voltage by current. This results in a surface impedance per unit length of:

$$
Z_{s}=\left(\frac{\delta}{w}\right) \frac{\gamma \operatorname{Re}(\gamma)}{\sigma}
$$

Recognizing $\delta w$ as the geometric factor $\Delta g$ for a parallelplate structure, we write:

$$
Z_{s}=\Delta g \frac{\gamma \operatorname{Re}(\gamma)}{\sigma}=j \omega \Delta L
$$

where $\Delta L=L^{\prime}-L$ is the added inductance from (7) and therefore:

$$
\mu_{\text {eff }}=\frac{\gamma \operatorname{Re}(\gamma)}{j \omega \sigma}=\mu_{\text {voigt }}+j\left|\mu_{\text {voigt }}\right|
$$

Knowing $\mu_{\text {eff }}$, we refer to (7) and hence we now have an expression for the complex inductance (containing $L$ and 
$R$ ) per unit length. The capacitance per unit length is found via (2) and (3). The shunt conductance per unit length is easily found from the capacitance (given a known dielectric loss tangent) by considering a complex $\varepsilon_{r}$ in (2)this is not described in detail in this report.

Now that all four circuit parameters, $R, L, C$, and $G$, are known, the impedance and propagation constant of the line can be easily determined [9] and thus the complex scattering-parameter matrix as a function of frequency can be simulated [11].
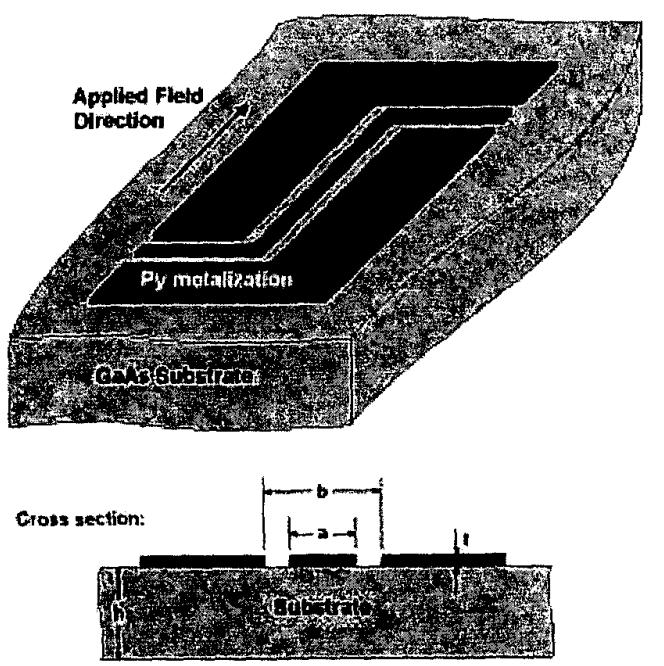

Fig. 1. Schematic of the CPW structure measured. Note the right-angle bends to allow probing from the sides and the applied field direction.

\section{RESULTS}

Fig. 1 shows the general construction of the line used for the measurements and simulations. The two rightangle bends allow the network analyzer probes to contact the line from either side while an electromagnet applies a field in the direction shown. The spacing between ground planes (b) is $148 \mu \mathrm{m}$ and the width of the center signal line (a) is $64 \mu \mathrm{m}$. In our previous study, the structure also included a narrower region that was designed to increase the magnetic effects: this region has a larger skin depth relative to the conductor widths. For the material

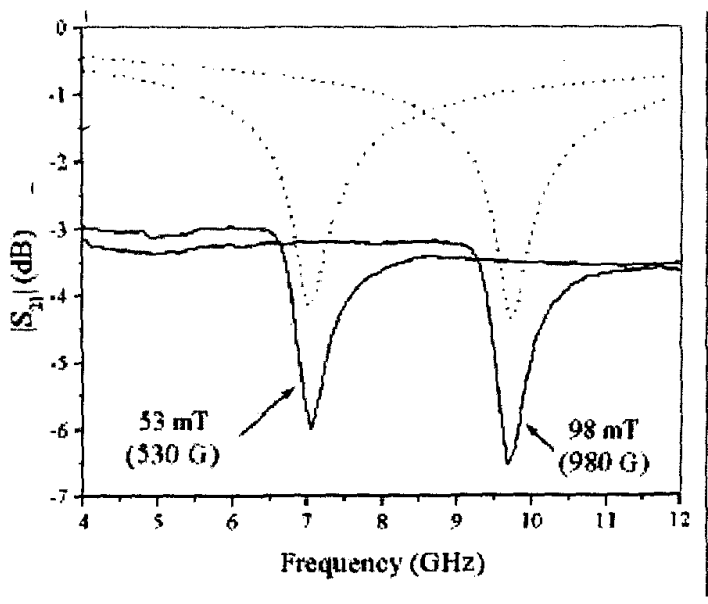

Fig. 2. Comparison between transmitted voltage magnitude experiment (solid lines) and simulation (dotted lines) for two different applied field values.

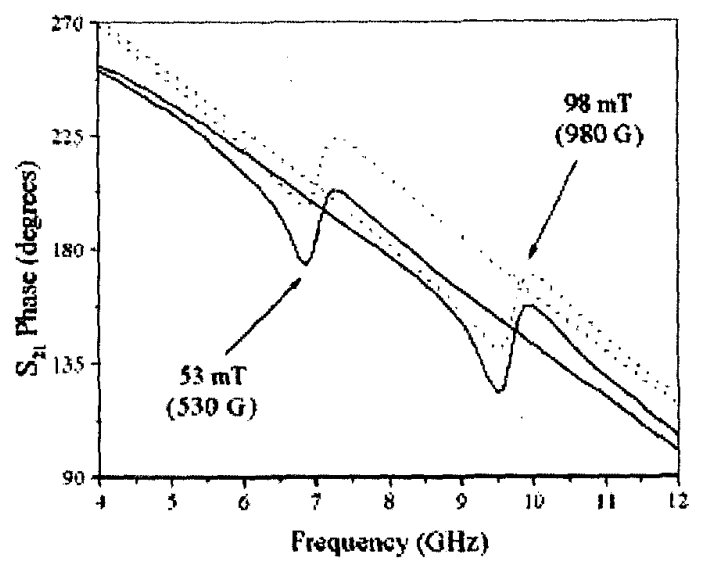

Fig. 3. Comparison between transmitted voltage phase experiment (solid lines) and simulation (dotted lines) for two different applied fields.

parameters, we used $850 \mathrm{kA} / \mathrm{m}$ for $M_{8}, 180 \mathrm{GHz} / \mathrm{T}$ for $\gamma_{0}$ and $6.25 \times 10^{8} \Omega^{-1} \mathrm{~m}^{-1}$ for $\sigma$.

Fig. 2 presents results demonstrating the tunable notch filter effect. Note that the solid experimental lines differ from the dotted simulation lines primarily because of additional background attenuation measured in the experiment. Note that, as expected, the notch frequency increases with applied field in accordance with the FMR frequency equation:

$$
\omega=\gamma_{0} \sqrt{B_{0}\left(B_{0}+\mu_{0} M_{S}\right)}
$$

where $\gamma_{0}$ is the gyromagnetic ratio, $B_{0}$ is the applied field, and $M_{\mathrm{s}}$ is the saturation magnetization. The notch depth is 
about $3 \mathrm{~dB}$ in voltage terms for a line length of $6.565 \mathrm{~mm}-$ this converts to about $10 \mathrm{~dB} / \mathrm{cm}$.

The phase shift effect occurs near the FMR frequency, as shown in Fig. 3. By changing the applied field, the phase of the transmitted wave can be increased or decreased continuously. For example, at $10 \mathrm{GHz}$ the phase can be increased by about 15 degrees by increasing the field and moving the resonance frequency closer to 10 $\mathrm{GHz}$. Considering that the line length is $6.565 \mathrm{~mm}$, this translates to a phase-shift of about 20 degrees $/ \mathrm{cm}$. This effect is well approximated in the simulation (dotted lines), except that the experimental lines (solid lines) are shifted down by about 10 degrees.

\section{CONCLUSION}

We present a simple model for CPW structures constructed of ferromagnetic conductors on a dielectric substrate. Unlike transmission lines with magnetic dielectrics, these structures demonstrate magnetic effects that occur only in the skin depth region of the conductive elements. Hence, we modify the circuit elements to account for skin depth and develop an expression for surface impedance that includes the magnetic effects. The result is a method for determining the impedance and propagation constant, given device geometry and magnetic properties. Our simulation of Py-based CPW lines approximates the magnitude and frequency position of the effects observed in our experimental measurements.

Transmission line structures based on ferromagnetic metals hold great promise for high-frequency applications. Our recent demonstrations of filters and phase-shifters based on these materials, along with this presentation of a model, increases the potential for future practical applications.

\section{ACKNOWLEDGEMENT}

This work was supported by ARO grants DAAD19-00-10146 and DAAG55-98-0294. We thank the authors of the
"MultiCal" software, which was used for TRL calibration. Finally, the authors thank Zbigniew Celinski and Robert Camley for theoretical and practical guidance.

\section{REFERENCES}

[1] W. S. Ishak, "Magnetostatic wave technology: a review," Proc. IEEE, vol. 72, pp. 171-87, 1988

[2] Ernst Schloemann, Randal Tustison, Jehoshua Weissman, H. Jerrold Van Hook, and Thomas Varitimos, "Epitaxial Fe films on GaAs for hybrid semiconductor-magnetic memories," J. Appl. Phys.; vol. 63, pp. 3140-2, 1988

[3] R. E. Camley and D. L. Mills, "Theory of microwave propagation in dielectric/magnetic film multilayer structures," J. Appl. Phys., vol. 82, pp. 3058-67, 1997

[4] N. Cramer, D. Lucic, R. E. Camley, and Z. Celinski, "High attenuation tunable microwave notch filters utilizing ferromagnetic resonance," J. Appl. Phys., vol. 87, pp. 6911 3,2000

[5] V. S. Liau, T. Wong, W. Stacey, S. Ali, and E. Schloemann, "Tunable band-stop filter based on epitaxial Fe film on GaAs," IEEE MTT-S, vol. 3, pp. 957-60, 1991

[6] C. S. Tsai, Jun Su, and C. C. Lee, "Wideband electronically tunable microwave bandstop filters using iron film-gallium arsenide waveguide structure," IEEE Trans. Magn., vol. 35, pp. 3178-80, 1999

[7] N. Cramer, D. Lucic, D. K. Walker, R. E. Camley and Z. Celinski, "Microwave tunable filters and phase-shifters based on transmission lines incorporating ferromagnetic metals," Submitted to IEEE Trans. Magn.

[8] B. C. Wadell, Transmission Line Design Handbook, Boston: Artech House, 1991.

[9] F. T. Ulaby, Fundamentals of Applied Electromagnetics, Upper Saddle River: Prentice Hall, 1999.

[10] R. J. Astalos and R. E. Camley, "Theory of a high frequency magnetic tunable filter and phase shifter," J. Appl. Phys., vol. 83, pp. 3744-9, 1998

[11] R. B. Marks and D. F. Williams, "A General Waveguide Circuit Theory," J. Res. NIST, vol. 5, pp. 533-62, 1992. 